\title{
Sex, deep sequencing and microRNAs
}

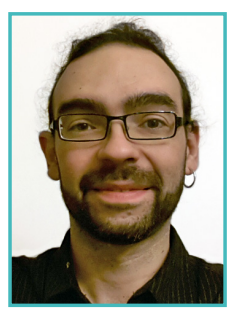

\section{Antonio Marco}

University of Essex, Essex, United Kingdom

Marco A (2014) EMBnet.journal 20(Suppl A), e765. http://dx.doi.org/10.14806/ej.20.A.765

Expression analyses often quantify gene product levels in samples under diverse conditions, from multiple tissues or from different developmental times. These analyses frequently miss an important aspect in many species: sex. Most animals and some plants have separate sexes, whose morphological differences are the outcome of the biased expression of some genes.

MicroRNAs are important regulators that repress translation by targeting gene transcripts. Recently, we have investigated microRNAs with sex-biased expression pattern from deep sequencing data. We found that sex-biased microRNAs (SBMiR) are, as expected, expressed in the gonads. Strikingly, the evolutionary dynamics of SBMiR is different to that of protein coding genes (SBPG). For instance, SBMiR are frequently locat- ed in the sex chromosomes, whilst SBPG tend to be in the autosomes. Interestingly, SBPG often have duplicated copies with no expression bias, and SBMiR usually arise de novo during evolution (no duplication).

In this talk I will explore these and other findings on sex-biased microRNAs, and why we must account for sex differences when planning expression profile experiments.

\section{References}

Marco A (2014) Sex-biased expression of microRNAs in Drosophila melanogaster. Open Biol 4, 140024. http://. dx.doi.org/10.1098/rsob.140024

Marco A, Kozomara A, Hui JHL, Emery AM, Rollinson D et al (2013) Sex-Biased Expression of MicroRNAs in Schistosoma mansoni. PloS Negl Trop Dis 7(9), e2402. http://dx.doi. org/10.1371/journal.pntd. 000240? 